\title{
Hereditarily indecomposable tree-like continua, II
}

\author{
by \\ W. T. Ingram (Houston, Tex.)
}

\begin{abstract}
In this paper is presented an example of an hereditarily indecomposable tree-like continuum such that each non-degenerate subcontinuum of it has positive span.
\end{abstract}

1. Introduction. In an abstract in 1951 Anderson [1] stated that there is an hereditarily indecomposable tree-like continuum which contains only degenerate chainable continua. In 1959 Anderson and Choquet [2] constructed a tree-like continuum in the plane such that no two of its non-degenerate subcontinua are homeomorphic. In 1967 Cook [3] constructed a continuum with the property that the identity is the only non-constant mapping of the continuum into itself. The purpose of this paper is to present an example of an hereditarily indecomposable tree-like continuum containing only degenerate subcontinua with span zero. Thus, the example of this paper contains only degencrate chainable continua. The construction of this continuum (in Section 5) is similar to that employed by Cook and the reader familiar with that paper will notice arguments here which are quite similar to those in that paper.

Throughout this paper the term mapping means continuous function and the term continuum means compact, connected metric space.

2. Atomic maps, span, and atriodic continua. In this section we establish the main lemmas for the constructions of this paper.

Defintion. Suppose $f$ is a mapping of a continuum $X$ into a continuum $Y$. The statement that $f$ is atomic means if $K$ is a subcontinuum of $X$ such that $f(K)$ is non-degenerate then $K=f^{-1}(f(K))$. (In [3, p. 242] Cook called such a map preatomic, but in [5, Theorem 1] Emeryk and Horbanowicz showed that such mappings are monotone making the usage here consistent with that of Anderson and Choquet $[2, \mathrm{p} .347]$.) The span of $f$, denoted $\sigma f$, is the least upper bound of the set to which the number $\varepsilon$ belongs if and only if there exists a subcontinuum $Z$ of $X \times X$ such that $p_{1}(Z)=p_{2}(Z)\left(p_{1}\right.$ and $p_{2}$ denote the two projections of $X \times X$ onto $X)$ and if $(x, y)$ is in $Z$ then $d(f(x), f(y)) \geqslant \varepsilon$. The span of $X$, denoted $\sigma X$, is the span of the identity mapping of $X$ onto $X$. 
THeOREM 1. Suppose $X$ is a continuum and $f$ is an atomic mapping of $X$ onto an atriodic continuum $Y$. If, for each point $y$ of $Y, f^{-1}(y)$ is atriodic, then $X$ is atriodic.

Proof. Suppose $H$ is a triod lying in $X$. Then $H$ is the union of three continua $H_{1}, H_{2}$, and $H_{3}$ such that the common part of each two of them is the common part of all three of them and is a proper subcontinuum of each one of them. By hypothesis $f(H)$ is non-degenerate.

We now show $f\left(H_{i}\right)$ is not $f(H)$ for $i=1,2,3$. If $i$ is an integer such that $f\left(H_{i}\right)$ is $f(H)$ then $f\left(H_{i}\right)$ is non-degenerate and $f^{-1}\left(f\left(H_{i}\right)\right)=H_{i}$. However, in $H_{j}, j \neq i$, there is a point $Q_{j}$ not in $H_{i}$. It is easy to see that that $f\left(Q_{j}\right)$ is not in $f\left(H_{i}\right)$.

Further, if $H_{i}$ and $H_{j}$ are two of $H_{1}, H_{2}$, and $H_{3}$, then $f\left(H_{i}\right) \cup f\left(H_{j}\right)$ is not $f(H)$. To see this, for convenience suppose $f\left(H_{1}\right) \cup f\left(H_{2}\right)$ is $f(H)$. Since $f\left(H_{1} \cup H_{2}\right)=f(H)$ and $f(H)$ is non-degenerate, $f^{-1} f\left(H_{1} \cup H_{2}\right)=H_{1} \cup H_{2}$. But, there is a point $Q_{3}$ of $H_{3}$ not in $H_{1} \cup H_{2}$ and $f\left(Q_{3}\right)$ is not in $f\left(H_{1}\right) \cup f\left(H_{2}\right)$.

Since $f(H)$ is the union of three continua, $f\left(H_{1}\right), f\left(H_{2}\right)$, and $f\left(H_{3}\right)$, such that no one of them is a subset of the union of the other two, $f(H)$ contains a triod [12]. This involves a contradiction.

THEOREM 2. If $X$ is a continuum, $f$ is a mono tone mapping of $X$ onto a continuium $Y$, and $\varepsilon$ is a positive number such that $\sigma Y>\varepsilon$, then $\sigma X>0$ and $\sigma f>\varepsilon$.

Proof. Suppose $\varepsilon$ is a positive number such that $\sigma Y>\varepsilon$. Since $f$ is uniformly continuous, there is a positive number $\delta$ such that if $(x, y)$ is a point of $X \times X$ and $d(x, y)<\delta$ then $d(f(x), f(y))<\varepsilon$.

We now show $\sigma X \geqslant \delta$. There is continuum $Z$ in $Y \times Y$ such that $p_{1}(Z)=p_{2}(Z)$ and if $(P, Q)$ is a point of $Z, d(P, Q) \geqslant \varepsilon$. Let $Z^{\prime}=(f \times f)^{-1}(Z)$ and note that $Z^{\prime}$ is a continuum because $f$ is monotone.

To see that $p_{1}\left(Z^{\prime}\right)=p_{2}\left(Z^{\prime}\right)$, suppose $(x, y)$ is a point of $Z^{\prime}$. Then $(f(x), f(y))$ is in $Z$. Since $p_{1}(Z)=p_{2}(Z)$, there are points $P$ and $Q$ of $Y$ such that $(P, f(x))$ and $(f(y), Q)$ are in $Z$. If $s$ and $t$ are points of $X$ such that $f(s)=P$ and $f(t)=Q$, then $(s, x)$ and $(y, t)$ are in $Z^{\prime}$.

If $(x, y)$ is in $Z^{\prime}$, then $d(x, y) \geqslant \delta$; for if $d(x, y)<\delta$ then $d(f(x), f(y))<\varepsilon$, but $(f(x), f(y))$ is in $Z$. Since $f \times f\left(Z^{\prime}\right)=Z, \sigma f>\varepsilon$.

3. A "spiral" to a simple triod. The crucial difference between the construction of this paper and that of Cook [3] is our need here to have a "spiral" to a simple triod such that the mapping $f$ of $T$ onto $T$ (see below for a definition of $f$ ) will extend to a mapping of the closure of the "spiral" to itself. The mapping $f$ does not extend to the usual (straight around) spiral to a simple triod. In this section we construct a "double spiral" to a simple triod which we will employ in the main construction of this paper.

We recall some definitions and notations from [7]. Denote by $T$ the set of all points $(Q, \theta)$ in the plane in polar coordinates such that $0 \leqslant \varrho \leqslant 1$ and $\theta$ is in $\left\{0, \frac{1}{2} \pi, \pi\right\}$. Denote by $A$ the point $\left(1, \frac{1}{2} \pi\right)$, by $B$ the point $(1, \pi)$, by $C$ the point $(1,0)$, and by $O$ the point $(0,0)$.
Denote by $f$ the mapping of $T$ onto $T$ defined as follows:

$$
f(\varrho, \theta)=\left\{\begin{array}{lll}
(1-2 \varrho, \pi) & \text { if } \quad 0 \leqslant \varrho \leqslant \frac{1}{2} \text { and } \theta=0, \\
(2 \varrho-1,0) & \text { if } \frac{1}{2} \leqslant \varrho \leqslant 1 \text { and } \theta=0, \\
(1-3 \varrho, \pi) & \text { if } \quad 0 \leqslant \varrho \leqslant \frac{1}{3} \text { and } \theta=\pi, \\
\left(3 \varrho-1, \frac{1}{2} \pi\right) & \text { if } \frac{1}{3} \leqslant \varrho \leqslant \frac{1}{2} \text { and } \theta=\pi, \\
\left(2-3 \varrho, \frac{1}{2} \pi\right) & \text { if } \frac{1}{2} \leqslant \varrho \leqslant \frac{2}{3} \text { and } \theta=\pi, \\
(3 \varrho-2), 0) & \text { if } \frac{2}{3} \leqslant \varrho \leqslant 1 \text { and } \theta=\pi, \\
(1-4 \varrho, \pi) & \text { if } \quad 0 \leqslant \varrho \leqslant \frac{1}{4} \text { and } \theta=\frac{1}{2} \pi, \\
\left(4 \varrho-1, \frac{1}{2} \pi\right) & \text { if } \frac{1}{4} \leqslant \varrho \leqslant \frac{1}{2} \text { and } \theta=\frac{1}{2} \pi, \\
\left(3-4 \varrho, \frac{1}{2} \pi\right) & \text { if } \frac{1}{2} \leqslant \varrho \leqslant \frac{3}{4} \text { and } \theta=\frac{1}{2} \pi, \\
(4 \varrho-3,0) & \text { if } \frac{3}{4} \leqslant \varrho \leqslant 1 \text { and } \theta=\frac{1}{2} \pi .
\end{array}\right.
$$

Denote by $M$ the inverse limit of the inverse limit sequence $\left\{T_{i}, f_{i}\right\}$ where, for each $i, T_{i}=T$ and $f_{i}=f$. In [7] the author showed that $M$ is an atriodic tree-like continuum with positive span.

Denote by $K$ the composant of $M$ containing the point $(C, C, C, \ldots)$. Note that $K$ is a topological ray, and let $h$ denote a homeomorphism throwing $(0,1]$ onto $K$. Then $h(1)$ is $(C, C, C, \ldots)$. Denote by $R$ the subset of $M \times[-1,1]$ to which the point $(x, y)$ belongs if and only if it is true that if $y$ is 0 then $x$ is in $M$, if $y>0$ then $x$ is $h(y)$, and if $y<0$ then $x$ is $h(-y)$. This is a "double spiral" to $M$. Denote by $F$ the mapping of $M$ onto $M$ induced by $f$, i.e. $F\left(x_{1}, x_{2}, \ldots\right)=\left(f\left(x_{1}\right), x_{1}, x_{2}, \ldots\right)$. Note that $G$, defined as follows is a mapping of $R$ onto $R$ :

$$
G(x, y)=\left\{\begin{array}{lll}
(F(x), 0) & \text { if } y=0, \\
\left(F(x), h^{-1} F h(y)\right) & \text { if } y>0, \\
\left(F(x),-h^{-1} F h(-y)\right) & \text { if } y<0 .
\end{array}\right.
$$

Denote by $S$ the "spiral" to $T, R / \pi_{1}\left(\pi_{1}\right.$ is the projection of $M$ onto $\left.T_{1}\right)$. The mapping $G$ of $R$ onto $R$ induces a mapping $\varphi$ of $S$ onto $S[4$, p. 126] and $\varphi \mid T$ is $f$.

4. The main constructions. In this section we describe the inverse limit systems that produce the main examples of the paper.

Defrnitions. If $f$ is a mapping of the continuum $X$ onto the continuum $Y$, $f$ is called an $A^{*}$-map if it is atomic and there do not exist infinitely many points $y$ of $Y$ such that $f^{-1}(y)$ is non-degenerate.

THEOREM 3. There exists an inverse limit sequence $\left\{X_{i}, f_{i}\right\}$ such that (1) $X_{1}$ is $T$ and, for each positive integer $n, X_{n}$ is a continuum, (2) for each $n, f_{n}$ is an $A^{*}$-map, (3) if $x$ is in $X_{n}$ and $f_{n}^{-1}(x)$ is non-degenerate then $x$ is interior to an arc lying in $X_{n}$ and $f_{n}^{-1}(x)$ is a simple triod, (4) if $x$ is in $X_{n}$ and $f_{n}^{-1}(x)$ is non-degenerate and $\alpha$ is an arc containing $x$ in its interior such that $\alpha$ contains no other point with a non-degenerate inverse image, then $f_{n}^{-1}(\alpha)$ is homeomorphic to $S,(5)$ if $T$ is a maximal simple triod lying in $X_{n}$ then $f_{n}^{-1}(T)$ is not a simple triod, and (6) if $\alpha$ is an arc lying in $X_{n}$ then there exists an integer $m>n$ ruch that $\left(f_{n}^{m}\right)^{-1}(\alpha)$ is not an arc. 
We adopt the following notation used by Cook [3]. If $\alpha$ is an ordered pair $(i, j)$ of positive integers, denote $i$ by $n_{1}(\alpha)$, denote $j$ by $n_{2}(\alpha)$, denote the ordered pair $(i+1, j)$ by $\alpha^{*}$, and denote the ordered pair $(i, j+1)$ by $\alpha^{\prime}$. Let $D$ denote the set all ordered pairs of positive integers directed by the relation $<$ where $\alpha<\beta$ if and only if $\alpha$ and $\beta$ are two elements of $D$ such that either $n_{1}(\alpha)<n_{1}(\beta)$ or $n_{1}(\alpha)=n_{1}(\beta)$ and $n_{2}(\alpha)<n_{2}(\beta)$.

Recall from Section 3 that $M$ denotes the atriodic tree-like continuum with positive span from [7].

THEorem 4. There exists an inverse limit sequence $\left\{X_{i}, f_{i}\right\}$ such that, (1) $X_{1}$ is $M$ and for each $n, X_{n}$ is an atriodic tree-like continuum with $\sigma X_{n}>0$, (2) for each $n$ and $m$ with $n<m, f_{n}^{m}$ is an atomic mapping and if $x$ is in $X_{i}$ where $i$ is a positive integer then $\left(f_{i}\right)^{-1}(x)$ is either degenerute or homeomorphic to $M$, and (3) if $n$ is a positive integer and $I$ is an arc lying in $X_{n}$ then there exists an integer $m>n$ such that $\left(f_{n}^{m}\right)^{-1}(I)$ contains a homeomorphic copy of $M$.

Proof. There exists an inverse mapping system $\left\{T_{\alpha}, \pi_{\alpha}^{\beta}\right\}$ over $D$ such that, (1) for each $\alpha$ in $D, T_{\alpha}$ is a continuum, (2) if $\alpha_{1}, \alpha_{2}, \ldots, \alpha_{n}$ is a sequence of elements of $D$ such that, for each $i<n, \alpha_{i+1}$ is either $\alpha_{i}^{*}$ or $\alpha_{i}^{\prime}$ then $\pi_{\alpha_{1}}^{\alpha_{n}}$ is the composite $\pi_{\alpha_{1}}^{\alpha_{2}} \circ \pi_{\alpha_{2}}^{\alpha_{3}} \circ \ldots \circ \pi_{\alpha_{n-1}}^{\alpha_{n}}$, (3) if $\alpha$ is in $D, T_{\alpha}$ contains only finitely many mutually exclusive simple triods, (4) if $\alpha$ is in $D$ and $n_{1}(\alpha)=1$ then $T_{\alpha}$ is a simple triod and $\pi_{\alpha}^{\alpha^{\prime}}$ is $f$, (5) for each positive integer $i$, then inverse limit sequence $\left\{T_{(n, i)}, \pi_{(n, i)}^{(m, i)}\right\}$ satisfies all the conditions of Theorem 3, and (6) if $n$ is a positive integer and $K$ is a maximal simple triod in $T_{(n, j)}$ which is thrown by $\pi_{(n, i)}^{(n, j)}$ onto a simple triod in $T_{(n, i)}$ then $\pi_{(n, i)}^{(n, j)} \mid K$ is $f^{j-i}$.

For each $n$ let $X_{n}$ be the inverse limit of the inverse limit sequence $\left\{T_{(n, i)}, \pi_{(n, i)}^{(n, j)}\right\}$ and let $f_{n}$ be the mapping of $X_{n+1}$ onto $X_{n}$ induced by $\pi_{(n, 1)}^{(n+1,1)}, \pi_{(n, 2)}^{(n+1,2)}, \pi_{(n, 3)}^{(n+1,3)}, \ldots$ Cook [3, Theorem 1, p. 242] has shown that $f_{n}$ is atomic, thus $f_{n}^{m}$ is atomic if $m>n$. Each $X_{n}$ is atriodic by Theorem 1 . By (6), for each $n, X_{n}$ contains a copy of $M$. Thus, for each $n, X_{n}$ has positive span.

THEOREM 5. The inverse limit of an inverse limit sequence satisfying the conditions of Theorem 4 is an atriodic tree-like continuum containing only degenerate subcontinua with span zero.

Proof. Such an inverse limit is atriodic because an inverse limit with each factor space an atriodic continuum is atriodic. Suppose $K$ is a non-degenerate subcontinuum of the inverse limit. By condition (3) of Theorem 4, some projection of $K$ contains a copy of $M$. This projection is atomic [3, Theorem 2, p. 242] so by Theorem $2 K$ contains a subcontinuum with positive span. Thus $K$ has positive span.

In the final section of this paper it is shown that each member of the collection $H$ defined in [10] has positive span. Denote by $N$ the member of the collection $H$ which is obtained by using an inverse limit on simple triods with odd numbered bonding maps "crooked" and even numbered bonding maps the mapping $f$ of section 3 of this paper.

THBoRem 6. There exists an inverse mapping sequence $\left\{Y_{i}, g_{i}\right\}$ such that, (1) $Y_{1}$ is $N$ and for each $i, Y_{i}$ is an hereditarily indecomposable tree-like continuum with positive span, (2) for each $i, g_{i}$ is an atomic mapping of $Y_{i+1}$ onto $Y_{i}$, and, if $y$ is a point of $Y_{i}, g_{i}^{-1}(y)$ is either degenerate or is homeomorphic to $N$, (3) if $i$ is a positive integer and $P$ is a pseudo-arc lying in $Y_{i}$ then there is an integer $j>i$ such that $\left(g_{i}^{j}\right)^{-1}(P)$ contains an hereditarily indecomposable tree-like continuum with positive span.

Proof. We wish to construct an inverse mapping system $\left\{T_{\alpha}, \pi_{\alpha}^{\beta}\right\}$ over $A$ as in the proof of Theorem 4 except that if we restrict our attention to an inverse limit sequence $\left\{T_{\gamma}, \pi_{\gamma}^{\delta}\right\}$ such that $n_{1}(\gamma)=n_{1}(\delta)=i$ then the inverse limit of this sequence is hereditarily indecomposable. This can be achieved by inserting sufficiently crooked mappings into the inverse mapping system of Theorem 4, so that each mapping of a maximal simple triod onto a simple triod is crooked in the manner described in [10]. It should be noted that such a crooked mapping of a simple triod extends to a crooked mapping of $S$ onto $S$. Let $Y_{i}$ be the inverse limit of the sequence $\left\{T_{\gamma}, \pi_{\gamma}^{\delta}\right\}$ where $n_{1}(\gamma)=n_{1}(\delta)=i$ and $g_{i}$ the mapping of $Y_{i+1}$ onto $Y_{i}$ induced by $\pi_{(i, 1)}^{(i+1), 1)}, \pi_{(i, 2)}^{(i+1,2)}$, $\pi_{(i, 3)}^{(i+1,3)}, \ldots$ Using again the result of Cook [3, Theorem 1, p. 242] $g_{i}$ is atomic. Since for each $i, Y_{i}$ contains a copy of $N, Y_{i}$ has positive span.

THEOREM 7. The inverse limit of an inverse limit sequence satisfying the conditions of Theorem 6 is an hereditarily indecomposable tree-like continuum containing only degenerate continua with span zero.

Proof. Such an inverse limit is hereditarily indecomposable because any inverse limit on hereditarily indecomposable continua is hereditarily indecomposable. That each non-degenerate subcontinuum has positive span may be seen using condition (3) of Theorem 6, the fact that the projections are atomic [3, Theorem 2, p. 242] and Theorem 2 as in the proof of Theorem 5 .

5. Span. In this section we prove that each of the continua in the collection $H$ defined in [10] has positive span. The proof is similar in nature to the proof that the continuum $M$ of [7] has positive span. However, so much modification of the argument has been made that a complete argument is given here.

The function $f$ was defined in Section 3 of this paper. The homeomorphism $r$ of $T$ onto $T[8]$ is defined as follows:

$$
r(\varrho, \theta)=\left\{\begin{array}{lll}
\left(\varrho, \frac{1}{2} \pi\right) & \text { if } & \theta=\frac{1}{2} \pi \\
(\varrho, \pi) & \text { if } \quad \theta=0 \\
(\varrho, 0) & \text { if } \quad \theta=\pi
\end{array}\right.
$$

Notation. If $p$ and $q$ are positive integers and $p<q$, the point $\frac{p A}{q}$ denotes $\left(\frac{p}{q}, \frac{\pi}{2}\right), \frac{p B}{q}$ denotes $\left(\frac{p}{q}, \pi\right)$ and $\frac{p C}{q}$ denotes $\left(\frac{p}{q}, 0\right)$. The notation $\langle\alpha, \beta\rangle$ implies that $\langle\alpha, \beta\rangle$ is a subcontinuum of $T \times T$ such that $p_{1}(\langle\alpha, \beta\rangle)$ is $\alpha, p_{2}(\langle\alpha, \beta\rangle)$ is $\beta$ and $\alpha$ and $\beta$ are arcs in $T$. If $X$ and $Y$ are points of $T$, the arc from $X$ to $Y$ in $T$ is denoted by $X Y$.

Definition. A subset $Z$ of $T \times T$ is said to have property $L^{\prime}$ provided $Z$ is the 
union of twenty-four continua $\langle O A, B C\rangle,\langle B C, O A\rangle,\langle O A, O B\rangle,\langle O B, O A\rangle$, $\langle O B, A C\rangle,\langle A C, O B\rangle,\langle O C, A B\rangle,\langle A B, O C\rangle,\langle O A, O C\rangle,\langle O C, O A\rangle,\left\langle O \frac{A}{2}, B C\right\rangle$, $\left\langle B C, O \frac{A}{2}\right\rangle,\left\langle O B, \frac{A}{2} C\right\rangle,\left\langle\frac{A}{2} C, O B\right\rangle,\langle O B, O C\rangle,\langle O C, O B\rangle,\left\langle O C, \frac{A}{2} B\right\rangle$, $\left\langle\frac{A}{2} B, O C\right\rangle,\left\langle\frac{A}{2} A, \frac{A}{2} B\right\rangle,\left\langle\frac{A}{2} B, \frac{A}{2} A\right\rangle,\left\langle O \frac{A}{2}, \frac{A}{2} A\right\rangle,\left\langle\frac{A}{2} A, O \frac{A}{2}\right\rangle,\left\langle\frac{A}{2} A, \frac{A}{2} C\right\rangle$, and $\left\langle\frac{A}{2} C, \frac{A}{2} A\right\rangle$.

Lemma 1. Suppose $[a, b]$ and $[c, d]$ are arcs and $H$ and $K$ are subcontinua of $[a, b] \times[c, d]$. If both $a$ and $b$ belong to $p_{1}(H)$ and both $c$ and $d$ belong to $p_{2}(K)$, then $H$ and $K$ contain a common point.

Lemma 1 is used often in the proofs of Lemmas 2, 3, 4, and 5, below. It was also a useful tool in some of the proofs of [9]

LEMMA 2. If $Z$ is a subset of $T \times T$ with property $L^{\prime}$, then $Z$ is a continuum.

Proof. Each of $\langle A C, O B\rangle,\langle A B, O C\rangle,\langle O A, O B\rangle,\langle O A, O C\rangle$, and $\left\langle O \frac{A}{2}, B C\right\rangle$ intersects $\langle O A, B C\rangle$ (use Lemma 1) so the union of these six continua is a continuum, $C_{1}$. Each of $\langle B C, O A\rangle,\langle A B, O C\rangle,\langle O B, O A\rangle,\langle O B, O C\rangle,\left\langle B C, O \frac{A}{2}\right\rangle$, $\left\langle\frac{A}{2} B, \frac{A}{2} A\right\rangle$, and $\left\langle O B, \frac{A}{2} C\right\rangle$ intersects $\langle O B, A C\rangle$ so the union of these eight continua is a continuum, $C_{2}$. Each of $\langle B C, O A\rangle,\langle A C, O B\rangle,\langle O C, O A\rangle,\langle O C, O B\rangle$, $\left\langle\frac{A}{2} C, \frac{A}{2} A\right\rangle$, and $\left\langle O C, \frac{A}{2} B\right\rangle$ intersects $\langle O C, A B\rangle$ so the union of these seven continua is a continuum, $C_{3}$. Since $C_{1}$ intersects both $C_{2}$ and $C_{3}$, the union of these three continua is a continuum. Further, denote by $C_{i}^{\prime}(i=1,2,3)$ the continuum resulting from the union of all continua $\langle\alpha, \beta\rangle$ where $\langle\beta, \alpha\rangle$ is a subset of $C_{i}$. Then, $C_{1}^{\prime}$ intersects $C_{2}, C_{2}^{\prime}$ intersects $C_{3}$, and $C_{3}^{\prime}$ intersects $C_{1}$, so the union of $C_{1}, C_{2}, C_{3}, C_{1}^{\prime}, C_{2}^{\prime}$, and $C_{3}^{\prime}$ is a continuum. The continuum $\left\langle O_{2}^{A}, \frac{A}{2} A\right\rangle$ intersects $C_{2}$ at a point of $\left\langle\frac{A}{2} B, \frac{A}{2} A\right\rangle$ while $\left\langle\frac{A}{2} A, O \frac{A}{2}\right\rangle$ intersects $C_{2}^{\prime}$ at a point of $\left\langle\frac{A}{2} A,{ }_{2}^{A} B\right\rangle$, so $Z$ is a continuum

LEMMA 3. If $Z$ is a subcontinuum of $T \times T$ with property $L^{\prime}$, then there exists a subcontinuum $Z_{r}$ with property $L^{\prime}$ such that $r \times r\left(Z_{r}\right)=Z$.

Lemma 4. Suppose $k$ is a mapping of $T$ onto $T$ such that $k^{-1}(x)=\{x\}$ for $x$ in $\left\{O, \frac{A}{2}, A, B, C\right\}$, and $Z$ is a subcontinuum of $T \times T$ with property $L^{\prime}$. Then there exists a subcontinuum $Z_{k}$ of $T \times T$ with property $L^{\prime}$ such that $k \times k\left(Z_{k}\right)=Z$.
Proof. If $\alpha$ and $\beta$ are in $\left\{O A, O B, O C, O \frac{A}{2}, \frac{A}{2} A, A B, A C, B C, \frac{A}{2} B, \frac{A}{2} C\right\}$ then $k|\alpha \times k| \beta$ is a mapping of $\alpha \times \beta$ onto $\alpha \times \beta$ which is essential on the boundary, $S^{1}$, of $\alpha \times \beta$. Thus $k|\alpha \times k| \beta$ is not homotopic to a mapping $g$ of $\alpha \times \beta$ to $S^{1}$ which has the property that $g\left|S^{1}=(k|\alpha \times k| \beta)\right| S^{1}$. By a theorem of Mazurkiewicz [11, Theorem I, p. 328] $k|\alpha \times k| \beta$ is weakly confluent. For each continuum $\langle\alpha, \beta\rangle$ of the twenty-four whose union is $Z$ let $\langle\alpha, \beta\rangle^{\prime}$ be the component of $(k|\alpha \times k| \beta)^{-1}$ $(\langle\alpha, \beta\rangle)$ which is thrown by $k|\alpha \times k| \beta$ onto $\langle\alpha, \beta\rangle$. The union of the twenty-four continua thus obtained is the desired continuum $Z_{k}$.

LEMMA 5. If $Z$ is a subcontinuum of $T \times T$ with property $L^{\prime}$, then there exists a subcontinuum $Z_{f}$ of $T \times T$ with property $L^{\prime}$ such that $f \times f\left(Z_{f}\right)=Z$.

Proof. We adopt the notation used in [8] as follows: if $\langle t, u\rangle$ is a subcontinuum of $Z$ and $v$ and $w$ are arcs in $T$ such that $f \mid v$ is a homeomorphism throwing $v$ onto $t$ and $f \mid w$ is a homeomorphism throwing $w$ onto $u$ then $L=(f \mid v)^{-1} \times(f \mid w)^{-1}(\langle t, u\rangle)$ is a continuum thrown by $f \times f$ onto $\langle t, u\rangle$ such that $p_{1}(L)=v$ and $p_{2}(L)=w$. This continuum will be denoted by $L(\langle t, u\rangle, v, w)$

Let

$$
\begin{aligned}
\alpha_{1}= & L_{1}^{1}\left(\langle A B, O C\rangle, O \frac{A}{2}, \frac{2 B}{3} B\right) \cup L_{2}^{1}\left(\left\langle\frac{A}{2} A, \frac{A}{2} C\right\rangle, \frac{3 A}{8} \frac{A}{2}, \frac{B}{2} B\right) \cup \\
& \cup L_{3}^{1}\left(\left\langle\frac{A}{2} A, O \frac{A}{2}\right\rangle, \frac{3 A}{8} \frac{A}{2}, \frac{B}{2} \frac{2 B}{3}\right) \cup L_{4}^{1}\left(\left\langle\frac{A}{2} A, O \frac{A}{2}\right\rangle, \frac{A}{2} \frac{5 A}{8}, \frac{B}{2} \frac{2 B}{3}\right) \cup \\
& \cup L_{5}^{1}\left(\left\langle\frac{A}{2} A, O \frac{A}{2}\right\rangle, \frac{A}{2} \frac{5 A}{8}, \frac{B}{3} \frac{B}{2}\right) \cup L_{6}^{1}\left(\left\langle\frac{A}{2} A, \frac{A}{2} B\right\rangle, \frac{A}{2} \frac{5 A}{8}, O \frac{B}{2}\right) \cup \\
& \cup L_{7}^{1}\left(\langle A C, O B\rangle, \frac{A}{2} A, O \frac{B}{3}\right) \cup L_{8}^{1}\left(\langle A C, O B\rangle, \frac{A}{2} A, O \frac{C}{2}\right) \cup \\
& \cup L_{9}^{1}\left(\langle O A, O B\rangle, \frac{A}{2} \frac{3 A}{4}, O \frac{C}{2}\right) \cup L_{10}^{1}\left(\langle O A, O B\rangle, \frac{A}{4} \frac{A}{2}, O \frac{C}{2}\right) \cup \\
& \cup L_{11}^{1}\left(\langle O A, B C\rangle, \frac{A}{4} \frac{A}{2}, O C\right) \cup L_{12}^{1}\left(\langle A B, O C\rangle, O \frac{A}{2}, \frac{C}{2} C\right)
\end{aligned}
$$

With four exceptions, successive terms of the sequence $L_{1}^{1}, L_{2}^{1}, \ldots, L_{12}^{1}$ may be seen to intersect with the aid of Lemma 1 . For example $L_{1}^{1}$ and $L_{2}^{1}$ are continua lying in $O \frac{A}{2} \times \frac{B}{2} B, p_{1} L_{1}^{1}$ contains both $O$ and $\frac{A}{2}$ while $p_{2} L_{2}^{1}$ contains both $\frac{B}{2}$ and $B$. The exceptions to using Lemma 1 here are to see that $L_{3}^{1}$ intersects $L_{4}^{1}, L_{4}^{1}$ intersects $L_{5,}^{1}, L_{7}^{1}$ intersect $L_{8}^{1}$, and $L_{9}^{1}$ intersects $L_{10}^{1}$. There is a point $\left(A, P_{1}\right)$ in $\left\langle\frac{A}{2} A, O \frac{A}{2}\right\rangle$ so $\left(\frac{A}{2},\left(f \mid \frac{B}{2} \frac{2 B}{3}\right)^{-1}\left(P_{1}\right)\right)$ is in both $L_{3}^{1}$ and $L_{4}^{1}$. There is a point $\left(P_{2}, \frac{A}{2}\right)$ in 
$\left\langle\frac{A}{2} A, O \frac{A}{2}\right\rangle$ so $\left(\left(f \frac{A}{2} \frac{5 A}{8}\right)^{-1}\left(P_{2}\right), \frac{B}{2}\right)$ is in both $L_{7}^{1}$ and $L_{8}^{1}$ and there is a point $\left(P_{3}, B\right)$ in $\langle O A, O B\rangle$ which lifts to a point of both $L_{9}^{1}$ and $L_{10}^{1}$.

Let $\alpha_{2}=\alpha_{1}^{-1}, \alpha_{3}=L_{1}^{1} \cup L_{2}^{1} \cup \ldots \cup L_{7}^{1}$ and $\alpha_{4}=\alpha_{3}^{-1}$. Let

$$
\begin{aligned}
\alpha_{5}=\alpha_{4} & \cup L_{1}^{5}\left(\langle O C, A B\rangle, \frac{2 B}{3} B, O \frac{A}{2}\right) \cup L_{2}^{5}\left(\langle O C, O B\rangle, \frac{2 B}{3} B, O \frac{A}{4}\right) \cup \\
\cup & L_{3}^{5}\left(\langle O C, O B\rangle, \frac{2 B}{3} B, O \frac{C}{3}\right) \cup L_{4}^{5}\left(\left\langle\frac{A}{2} C, O B\right\rangle, \frac{B}{2} B, O \frac{C}{2}\right) \cup \\
\cup & \left.L_{5}^{5}\left(O \frac{A}{2}, B C\right\rangle, \frac{B}{2} \frac{2 B}{3}, O C\right) .
\end{aligned}
$$

By using Lemma 1 it can be seen that $L_{1}^{5}$ intersects $L_{2}^{5}, L_{3}^{5}$ intersects $L_{4}^{5}$, and $L_{4}^{5}$ intersects $L_{5}^{5}$. A point $\left(P_{5}, B\right)$ of $\langle O C, O B\rangle$ lifts to a point lying in both $L_{2}^{5}$ and $L_{3}^{5}$. Now, $\alpha_{4}$ contains $\left(L_{1}^{1}\right)^{-1}=L\left(\langle O C, A B\rangle, \frac{2 B}{3} B, O \frac{A}{2}\right)$ and since this is $L_{1}^{5}, \alpha_{5}$ is a continuum. Let $\alpha_{6}=\alpha_{5}^{-1}$.

Let

$$
\begin{aligned}
\alpha_{7}= & L_{1}^{7}\left(\left\langle O B, \frac{A}{2} C\right\rangle, O \frac{C}{2}, \frac{B}{2} B\right) \cup L_{2}^{7}\left(\left\langle B C, O \frac{A}{2}\right\rangle, O C, \frac{B}{2} \frac{2 B}{3}\right) \cup \\
& \cup L_{3}^{7}\left(\left\langle B C, O \frac{A}{2}\right\rangle, O C, \frac{B}{3} \frac{B}{2}\right) \cup L_{4}^{7}\left(\left\langle O C, \frac{A}{2} B\right\rangle, \frac{C}{2} C, O \frac{B}{2}\right) \cup \\
& \cup L_{5}^{7}\left(\langle O C, O B\rangle, \frac{C}{2} C, O \frac{B}{2}\right) \cup L_{6}^{7}\left(\langle O C, O B\rangle, \frac{C}{2} C, O \frac{A}{4}\right) \cup \\
& \cup L_{7}^{7}\left(\langle O C, A B\rangle, \frac{C}{2} C, O \frac{A}{2}\right) \cup L_{8}^{7}\left(\langle B C, O A\rangle, O C, \frac{A}{4} \frac{A}{2}\right) \cup \\
& \cup L_{9}^{7}\left(\langle O B, O A\rangle, O \frac{C}{2}, \frac{A}{4} \frac{A}{2}\right) \cup L_{10}^{7}\left(\langle O B, O A\rangle, O \frac{C}{2}, \frac{A}{2} \frac{3 A}{4}\right) \cup \\
& \cup L_{11}^{7}\left(\langle O B, A C\rangle, O \frac{C}{2}, \frac{A}{2} A\right) .
\end{aligned}
$$

By using Lemma 1 and the facts that $\left\langle B C, O \frac{A}{2}\right\rangle$ contains a point with second coordinate $\frac{A}{2},\langle O C, O B\rangle$ contains a point with second coordinate $B$, and $\langle O B, O A\rangle$ contains a point with second coordinate $A$, as before it can be seen that $\alpha_{7}$ is a continuum. Let $\alpha_{8}=\alpha_{7}^{-1}$ and let $\alpha_{9}=L_{7}^{7} \cup L_{8}^{7} \cup \ldots \cup L_{11}^{7}$ and $\alpha_{10}=\alpha_{9}^{-1}$.
Let

$$
\begin{aligned}
\alpha_{11}= & L_{1}^{11}\left(\langle A B, O C\rangle, O \frac{A}{2}, \frac{2 B}{3} B\right) \cup L_{2}^{11}\left(\left\langle\frac{A}{2} A, \frac{A}{2} C\right\rangle, \frac{3 A}{8} \frac{A}{2}, \frac{B}{2} B\right) \cup \\
& \cup L_{3}^{11}\left(\left\langle\frac{A}{2} A, O \frac{A}{2}\right\rangle, \frac{3 A}{8} \frac{A}{2}, \frac{B}{2} \frac{2 B}{3}\right) \cup L_{4}^{11}\left(\left\langle\frac{A}{2} A, O \frac{A}{2}\right\rangle, \frac{3 A}{8} \frac{A}{2}, \frac{B}{3} \frac{B}{2}\right) \cup \\
& \cup L_{5}^{11}\left(\left\langle\frac{A}{2} A, \frac{A}{2} B\right\rangle, \frac{3 A}{8} \frac{A}{2}, O \frac{B}{2}\right) \cup L_{6}^{11}\left(\langle O A, O B\rangle, \frac{A}{4} \frac{A}{2}, O \frac{B}{3}\right) \cup \\
& \cup L_{7}^{11}\left(\langle O A, O B\rangle, \frac{A}{4} \frac{A}{2}, O \frac{C}{2}\right) \cup L_{8}^{11}\left(\langle O A, B C\rangle, \frac{A}{4} \frac{A}{2}, O C\right)
\end{aligned}
$$

Here Lemma 1 and the facts that $\left\langle\frac{A}{2} A, O \frac{A}{2}\right\rangle$ contains a point with second coordinate $\frac{A}{2}$ and $\langle O A, O B\rangle$ contains a point with second coordinate $B$ may be used to see that $\alpha_{11}$ is a continuum. Let $\alpha_{12}=\alpha_{11}^{-1}$.

Let

$$
\begin{aligned}
\alpha_{13}= & L_{1}^{13}\left(\left\langle\frac{A}{2} B, O C\right\rangle, O \frac{B}{2}, \frac{C}{2} C\right) \cup L_{2}^{13}\left(\left\langle O \frac{A}{2}, B C\right\rangle, \frac{B}{3} \frac{B}{2}, O C\right) \cup \\
& \cup L_{3}^{13}\left(\left\langle O \frac{A}{2}, B C\right\rangle, \frac{B}{2} \frac{2 B}{3}, O C\right) \cup L_{4}^{13}\left(\left\langle\frac{A}{2} C, O B\right\rangle, \frac{B}{2} B, O \frac{C}{2}\right) \cup \\
& \cup L_{5}^{13}\left(\left\langle\frac{A}{2} C, O B\right\rangle, \frac{B}{2} B, O \frac{A}{4}\right) \cup L_{6}^{13}\left(\langle O C, A B\rangle, \frac{2 B}{3} B, O \frac{A}{2}\right)
\end{aligned}
$$

That $\alpha_{13}$ is a continuum may be seen with the aid of Lemma 1 and the facts that $\left\langle O \frac{A}{2}, B C\right\rangle$ contains a point with first coordinate $\frac{A}{2}$ and $\left\langle\frac{A}{2} C, O B\right\rangle$ contains a point with second coordinate $B$. Let $\alpha_{14}=\alpha_{13}^{-1}$ and $\alpha_{15}=L_{1}^{13} \cup L_{2}^{13} \cup L_{3}^{13} \cup L_{4}^{13}$ and $\alpha_{16}=\alpha_{15}^{-1}$

Let

$$
\alpha_{17}=\alpha_{16} \cup L_{1}^{17}\left(\left\langle O C, \frac{A}{2} B\right\rangle, \frac{C}{2} C, O \frac{3 A}{8}\right) \cup L_{2}^{17}\left(\langle O C, A B\rangle, \frac{C}{2} C, O \frac{A}{2}\right) .
$$

That $L_{1}^{17}$ intersects $L_{2}^{17}$ may be seen using Lemma 1 . Since $\left(L_{1}^{13}\right)^{-1}$ is a subset of $\alpha_{16},\left(L_{1}^{13}\right)^{-1}=L\left(\left\langle O C, \frac{A}{2} B\right\rangle, \frac{C}{2} C, O \frac{B}{2}\right)$, and $\left\langle O C, \frac{A}{2} B\right\rangle$ contains a point with second coordinate $B, L_{1}^{17}$ may be seen to intersect $\left(L_{1}^{13}\right)^{-1}$. Thus $\alpha_{17}$ is a continuum. Let $\alpha_{18}=\alpha_{17}^{-1}$. 
Let

$$
\begin{aligned}
\alpha_{19} & =L_{1}^{19}\left(\langle O C, A B\rangle, \frac{3 A}{4} A, O \frac{A}{2}\right) \cup L_{2}^{19}\left(\langle A C, O B\rangle, \frac{A}{2} A, O \frac{A}{4}\right) \cup \\
& \cup L_{3}^{19}\left(\langle A C, O B\rangle, \frac{A}{2} A, O \frac{B}{3}\right) \cup L_{4}^{19}\left(\left\langle\frac{A}{2} A, \frac{A}{2} B\right\rangle, \frac{A}{2} \frac{5 A}{8}, \frac{O B}{2}\right) \cup \\
& \cup L_{5}^{19}\left(\left\langle\frac{A}{2} A, O \frac{A}{2}\right\rangle, \frac{A}{2} \frac{5 A}{8}, \frac{B}{3} \frac{B}{2}\right) \cup L_{6}^{19}\left(\left\langle\frac{A}{2} A, O \frac{A}{2}\right\rangle, \frac{A}{2} \frac{5 A}{8}, \frac{B}{2} \frac{2 B}{3}\right) \cup \\
& \cup L_{7}^{19}\left(\left\langle\frac{A}{2} A, \frac{A}{2} C\right\rangle, \frac{A}{2} \frac{5 A}{8}, \frac{B}{2} B\right) .
\end{aligned}
$$

Since $\langle A C, O B\rangle$ contains a point with second coordinate $B$ and $\left\langle\frac{A}{2} A, O \frac{A}{2}\right\rangle$ contains a point with second coordinate $\frac{A}{2}$, it may be seen that $L_{2}^{19}$ intersects $L_{3}^{19}$ and $L_{5}^{19}$ intersects $L_{6}^{19}$. Thus, by using Lemma $1, \alpha_{19}$ is a continuum. Let $\alpha_{20}=\alpha_{19}^{-1}$. Let $\alpha_{21}=L_{1}^{19} \cup L_{2}^{19}$ and $\alpha_{22}=\alpha_{21}^{-1}$.

Let

$$
\alpha_{23}=\alpha_{21} \cup L_{1}^{23}\left(\langle A C, O B\rangle, \frac{A}{2} A, O \frac{C}{2}\right) \cup L_{2}^{23}\left(\langle O A, B C\rangle, \frac{A}{2} \frac{3 A}{4}, O C\right) .
$$

Using Lemma $1, L_{1}^{23}$ intersects $L_{2}^{23}$. Since $\langle A C, O B\rangle$ contains a point with second coordinate $B$, it may be seen that $L_{2}^{19}$ intersects $L_{1}^{23}$ and so $\alpha_{23}$ is a continuum. Let $\alpha_{24}=\alpha_{23}^{-1}$.

Let $Z_{f}=\alpha_{1} \cup \alpha_{2} \cup \ldots \cup \alpha_{24}$. By Lemma $2 Z_{f}$ is a continuum. The continua $\alpha_{1}, \alpha_{2}, \ldots, \alpha_{24}$ are, in order, the continua required so that $Z_{f}$ have property $L^{\prime}$ By construction $f \times f\left(Z_{f}\right)=Z$.

Let $\mathscr{K}$ denote the set to which $k$ belongs if and only if $k$ is a mapping of $T$ onto $T$ such that $k^{-1}(x)=\{x\}$ for each point $x$ of $\left\{A, B, C, O, \frac{A}{2}\right\}$.

Lemma 6. Suppose, for each $n, f_{n}$ is in $\mathscr{K} \cup\{f, r\}$. Then, if $n>1, \sigma f_{1}^{n} \geqslant \frac{1}{2}$ where $f_{1}^{n}=f_{1} \cdot f_{2} \cdot \ldots \cdot f_{n-1}$.

\section{Proof. Let}

$$
\begin{aligned}
Z_{1}= & ((O A \times\{B\}) \cup(\{A\} \times B C)) \cup((B C \times\{A\}) \cup(\{B\} \times O A)) \cup \\
& \cup((O A \times\{B\} \cup(\{A\} \times O B)) \cup((O B \times\{A\}) \cup(\{B\} \times O A)) \cup \\
& \cup((O B \times\{A\}) \cup(\{B\} \times A C)) \cup((\{A\} \times O B) \cup(A C \times\{B\})) \cup \\
& \cup((O C \times\{A\}) \cup(\{C\} \times A B)) \cup((\{A\} \times O C) \cup(A B \times\{C\})) \cup \\
& \cup((O C \times\{A\}) \cup(\{C\} \times O A)) \cup((\{A\} \times O C) \cup(O A \times\{C\})) \cup \\
& \cup\left(\left(O \frac{A}{2} \times\{B\}\right) \cup\left(\left\{\frac{A}{2}\right\} \times B C\right)\right) \cup\left(\left(\{B\} \times O \frac{A}{2}\right) \cup\left(B C \times\left\{\frac{A}{2}\right\}\right)\right) \cup
\end{aligned}
$$

$$
\begin{aligned}
& \cup\left((O B \times\{C\}) \cup\left(\{B\} \times \frac{A}{2} C\right)\right) \cup\left((\{C\} \times O B) \cup\left(\frac{A}{2} C \times\{B\}\right)\right) \cup \\
& \cup((O B \times\{C\}) \cup(\{B\} \times O C)) \cup((\{C\} \times O B) \cup(O C \times\{B\})) \cup \\
& \cup\left((O C \times\{B\}) \cup\left(\{\dot{C}\} \times \frac{A}{2} B\right)\right) \cup\left((\{B\} \times O C) \cup\left(\frac{A}{2} B \times\{C\}\right)\right) \cup \\
& \cup\left(\left(\frac{A}{2} A \times\{B\}\right) \cup\{A\} \times \frac{A}{2} B\right) \cup\left(\left(\{B\} \times \frac{A}{2} A\right) \cup\left(\frac{A}{2} B \times\{A\}\right)\right) \cup \\
& \cup\left(\left(\frac{A}{2} A \times\{0\}\right) \cup\left(\{A\} \times O \frac{A}{2}\right)\right) \cup\left(\left(\{O\} \times \frac{A}{2} A\right) \cup\left(O \frac{A}{2} \times\{A\}\right)\right) \cup \\
& \cup\left(\left(\frac{A}{2} A \times\{C\}\right) \cup\left(\{A\} \times \frac{A}{2} C\right) \cup\left(\left(\{C\} \times \frac{A}{2} A\right) \cup\left(\frac{A}{2} C \times\{A\}\right)\right) .\right.
\end{aligned}
$$

If $(p, q)$ is in $Z_{1}$ then $d(p, q) \geqslant \frac{1}{2}$ and $Z_{1}$ has property $L^{\prime}$. The lemma follows by induction using Lemmas 3,4 , and 5 .

THeOREM 8. Suppose, for each $n, T_{n}$ is $T$ and $f_{n}$ is in $\mathscr{K} \cup\{r, f\}$ and $M$ is the inverse limit of the inverse limit sequence $\left\{T_{n}, f_{n}\right\}$. Then $\sigma M>0$.

Proof. Apply Lemma 6 and Theorem 4 of [7].

THEOREM 9. Each continum in the collection $H$ has positive span.

THEOREM 10. No continum in the collection $H$ is homogeneous.

Proof. Suppose $X$ is a member of $H$ which is homogeneous. In the proof that $X$ has positive span we show that for each positive integer $n$ there exists a subcontinuum $V_{n}$ of $T \times T$ such that $p_{1}\left(V_{n}\right)=O B$ and $p_{2}\left(V_{n}\right)=O C$ and $f_{1}^{n} \times f_{1}^{n}\left(V_{n}\right)$ is a subset of a continuum $Z_{1}$ in $T_{1} \times T_{1}$ with the property that $d(x, y) \geqslant \frac{1}{2}$ if $(x, y)$ is in $Z_{1}$. The continuum $X$ is the inverse limit of $\left\{T_{i}, \pi_{i}^{j}\right\}$ where $T_{i}=T$ and $\pi_{i}^{i+1}$ is in $\left\{k_{i} \cdots f, k_{i}, r \circ\right.$ with $k_{i}$ "crooked".

Since $X$ is homogeneous, by a theorem of Hagopian [6] there exists a positive number $\delta$ such that if $P$ and $Q$ are points of $X$ and $d(P, Q)<\delta$ then there is a homeomorphism $h$ of $X$ onto $X$ such that $h(P)=Q$ and if $x$ is a point of $X$ then $d(x, h(x))<\frac{1}{4}$. There exists a positive integer $N$ such that if $x$ and $y$ are points of $X$ such that $x_{N-1}=y_{N-1}$ then $d(x, y)<\delta$. Let $P$ and $Q$ be points of $X$ such that $P_{N}$ is $B$ and $Q_{N}$ is $C$. Then $P_{N-1}=Q_{N-1}$ so $d(P, Q)<\delta$. Let $h$ be a homeomorphism of $M$ onto $M$ such that $h(P)=Q$ and if $x$ is in $M, d(x, h(x))<\frac{1}{4}$. Consider a subcortinuum $K$ of $X$ containing $P$ such that $\pi_{N} K=[O B]$. Since $h(P)=Q, \pi_{N} h(K)$ contains $C$.

Case 1. If $\pi_{N} h(K)$ is a subset of $[O C]$, then $L=\{(x, y) \mid(x, y)$ is in $T \times T$ and there is a point $z$ of $K$ such that $x=\pi_{N}(z)$ and $\left.y=\pi_{N} h(z)\right\}$ is a continuum in $[O B] \times[O C]$ such that $p_{1} L=O B$ and $p_{2} L$ is a subset of $O C$. Thus, $L$ contains a point of $V_{N}$. This gives a point $z$ of $K$ such that $\left(\pi_{N}(z), \pi_{N} h(z)\right)$ is in $V_{N}$, so $\left(\pi_{1}(z), \pi_{1} h(z)\right)$ is in $Z_{1}$. This means $d(z, h(z)) \geqslant \frac{1}{4}$, a contradiction. 
Case 2. If $O C$ is a subset of $\pi_{N} h(K)$, there is a subcontinuum $K_{0}$ of $K$ such that $\pi_{N} h\left(K_{0}\right)=O C$. Then $L=\{(x, y)\}(x, y)$ is in $T \times T$ and there is a point $z$ of $K_{0}$ such that $x=\pi_{N}(z)$ and $\left.y=\pi_{N} h(z)\right\}$ is a subcontinuum of $T \times T$ such that $P_{1}(L)$ is a subset of $O B$ and $P_{2}(L)=O C$. Thus, $L$. contains a point of $V_{N}$. As before, this involves a contradiction.

\section{References}

[1] R. D. Anderson, Hereditarily indecomposable plane continua (Abstract), Bull. Amer. Math. Soc. 57 (1951), p. 185.

[2] - and G. Choquet, A plane continum no two of whose non-degenerate subcontimus are homeomorphic: an application of inverse limits, Proc. Amer. Math. Soc. 10 (1959), pp. 347 353.

[3] H. Cook, Continua which admit only the identity mapping onto non-degenerate sulucontinua, Fund. Math. 60 (1967), pp. 241-249.

[4] J. Dugundji, Topology, Allyn and Bacon, Inc., Boston 1966.

[5] A. Emeryk and Z. Horbanowicz, On atomic mappings, Collog. Math. 27 (1973), pp. 49-55.

[6] C. L. Hagopian, Homogeneous plane continut, Houston J. Math. 1 (1975), pp. 3541.

[7] W. T. Ingram, An atriodic tree-like continum with positive spant. Fund. Math. 77 (1972), pp. $99-107$.

[8] - An uncountable collection of mutually exclusive planar atriodic tree-like continua with positive span, Fund. Math. 85 (1974), pp. 73-78.

[9] - Concerning atriodic tree-like continua, Fund. Math. 101 (1978), pp. 189-193.

[10] - Hereditarily indecomposable tree-like continua, Fund. Math. 103 (1979), pp. 61 -64.

[11] S. Mazurkiewicz, Sur l'existence des continus indécomposables; Fund. Math. 25 (1935), pp. $327-328$.

[12] R. H. Sorgenfrey, Concerning triodic continua, Amer. J. Math, 66 (1944), pp. 439-460.

UNIVERSITY OF HOUSTON

Houston, Texas

\section{Convexity on a topological space}

by

\section{H. Komiya (Tokyo)}

Abstract. Although convexity is an attribute of subsets of linear spaces in general, we define convexity on topological spaces without linear structures paying attention to the concept of convex hull. Then some theorems which have been obtained in linear topological spaces are given in these spaces.

Takahashi [5] discussed a convexity on a metric space. In this paper, we discuss a convexity on a topological space without linear space structure. We introduce a convexity on a topological space and several concepts concerning the convexity, and obtain some theorems which generalize the theorems proved by Browder [1], Fan [2] and Sion [4]. All topological structures are implicitly assumed to satisfy Hausdorff separation axiom.

The author tenders his very warm thanks to Professor W. Takahashi for his advice in preparing this paper.

1. Definitions and some elementary properties. Let $X$ be a topological space, $\mathscr{A}(X)$ the family of all subsets of $X$ and $\mathscr{F}(X)$ the family of all finite subsets of $X$. An $H$-operator on $X$ is a mapping $\langle\cdot\rangle$ from $\mathscr{A}(X)$ into $\mathscr{A}(X)$ satisfying the following conditions:

(a) $\langle\varnothing\rangle=\varnothing$, where $\varnothing$ is the empty set;

(b) $\langle\{x\}\rangle=\{x\}, x \in X$;

(c) $\langle\langle A\rangle\rangle=\langle A\rangle, A \in \mathscr{A}(X)$;

(d) $\langle A\rangle=\bigcup\{\langle F\rangle: F \subset A, F \in \mathscr{F}(X)\}$.

The image $\langle A\rangle$ of $A$ is said to be the convex hull of $A$. A convex set in $X$ is a subset of $X$ which is equal to its convex hull.

Proposition 1. (i) An. $H$-operator is monotone, i.e. if $A \subset B$, then $\langle A\rangle \subset\langle B\rangle$.

(ii) The convex hull $\langle A\rangle$ of $A \in \mathscr{A}(X)$ is the smallest convex set containing $A$.

(iii) The entire space $X$ and the empty set $\varnothing$ are convex sets.

(iv) If $\left\{C_{v}\right\}_{v \in I}$ is a family of convex sets, then $\bigcap_{v \in I} C_{v}$ is a convex set.

(v) If $\left\{C_{v}\right\}_{v \in I}$ is a family of convex sets such that for any two indices $v_{1}$ and $v_{2}$ there exists an index $\mu$ with $C_{\mu} \subset C_{v_{1}} \cap C_{v_{2}}$, then $\bigcup_{v \in Y_{v}} C_{v}$ is a convex set. 